\title{
Inconsistency between Different Measures of Sexual Selection
}

\author{
Patrick S. Fitze ${ }^{1, \star}$ and Jean-François Le Galliard ${ }^{2}$
}

1. Department of Ecology and Evolution (DEE), University of Lausanne, Biophore, 1015 Lausanne, Switzerland; Department of Biodiversity and Evolutionary Biology (BEB), Museo Nacional de Ciencias Naturales (MNCN), Consejo Superior de Investigaciones Científicas (CSIC), C/José Gutiérrez Abascal 2, 28006 Madrid, Spain; and Instituto Pirenaico de Ecología (IPE), CSIC, Avenida Regimiento de Galicia s/n, 22700 Jaca (Huesca), Spain; 2. Écologie et Évolution, Université Pierre et Marie Curie, Unité Mixte de Recherche 7625, École Normale Supérieure (ENS), Centre National de la Recherche Scientifique (CNRS), 7 Quai St Bernard, 75005 Paris, France; and Centre de Recherche en Ecologie Expérimentale et Prédictive (CEREEP)-Ecotron IleDeFrance, Unité Mixte de Service 3194, ENS, CNRS, 78 rue du Château, 77140 St-Pierre-lès-Nemours, France

Submitted August 3, 2010; Accepted March 22, 2011; Electronically published June 29, 2011

Online enhancement: appendix. Dryad data: http://dx.doi.org/10.5061/dryad.8872.

ABSTRACT: Measuring the intensity of sexual selection is of fundamental importance to the study of sexual dimorphism, population dynamics, and speciation. Several indices, pools of individuals, and fitness proxies are used in the literature, yet their relative performances are strongly debated. Using 12 independent common lizard populations, we manipulated the adult sex ratio, a potentially important determinant of the intensity of sexual selection at a particular time and place. We investigated differences in the intensity of sexual selection, as estimated using three standard indices of sexual selection-the standardized selection gradient $\left(\beta^{\prime}\right)$, the opportunity of selection $(I)$, and the Bateman gradient $\left(\beta_{\mathrm{ss}}\right)$-calculated for different pools of individuals and different fitness proxies. We show that results based on estimates of $I$ were the opposite of those derived from the other indices, whereas results based on estimates of $\beta^{\prime}$ were consistent with predictions derived from knowledge about the species' mating system. In addition, our estimates of the strength and direction of sexual selection depended on both the fitness proxy used and the pool of individuals included in the analysis. These observations demonstrate inconsistencies in distinct measures of sexual selection and underscore the need for caution when comparing studies and species.

Keywords: standardized selection gradient, opportunity for sexual selection, Bateman gradient, natural selection, adult sex ratio, common lizard.

\section{Introduction}

Darwin was the first to understand the evolutionary importance of sexual selection (Darwin 1871). Nowadays, it is well established that sexual selection is responsible for the evolution of many secondary sexual characters and extraordinary behaviors (e.g., Andersson 1994) and plays an important role in population dynamics (Doherty et al. 2003; Le Galliard et al. 2005b). However, disagreement

* Corresponding author; e-mail: patrick.fitze@unil.ch.

Am. Nat. 2011. Vol. 178, pp. 256-268. (c) 2011 by The University of Chicago. 0003-0147/2011/17802-52352\$15.00. All rights reserved.

DOI: $10.1086 / 660826$ persists regarding the actual definition of sexual selection (e.g., Carranza 2009; but see Shuker 2010 for the widely accepted standard definition of sexual selection), leading to conflicting views on how and when to measure sexual selection (Kokko et al. 1999; Shuster and Wade 2003).

Several indices of sexual selection have been used to measure sexual selection, the most prevalent of which include the standardized selection gradient $\left(\beta^{\prime}\right.$; Lande and Arnold 1983), the opportunity of selection ( $I$; Crow 1958; Wade 1979; Wade and Arnold 1980; Shuster and Wade 2003; Jones et al. 2004), and the Bateman gradient ( $\beta_{\mathrm{ss}}$; Bateman 1948; Andersson and Iwasa 1996; Jones et al. 2004). $\beta^{\prime}$ measures realized sexual selection with respect to a specific trait using a regression of standardized fitness against standardized trait values and thus may assume a causal relationship between fitness and an investigated trait. As such, $\beta^{\prime}$ may fail to identify sexual selection on other unrelated traits. The opportunity of selection measures standardized variance in reproductive success $(I)$ or variance in mating success $\left(I_{s}\right)$, the latter being referred to as "opportunity of sexual selection." Both indices are derived using the same mathematical formula but differ in the fitness proxy used; $I_{\mathrm{s}}$ corresponds to the theoretical upper limit of the strength of sexual selection and also includes processes that generate random variance (e.g., Klug et al. 2010), and $I_{\mathrm{s}}$ is proposed to be a close correlate of total sexual selection-that is, sexual selection summed over all existing traits (e.g., Wade 1979). $\beta_{\text {ss }}$ is the slope of the linear relationship between reproductive success and mating success (for a detailed explanation, see Jones et al. 2004). It measures how success in mating translates into reproductive success (Wade 1979; Shuster and Wade 2003; Jones et al. 2004). The indices $I, I_{s}$, and $\beta_{\text {ss }}$ are independent of phenotypic traits and are believed to be more general measures of the strength of sexual selection than $\beta^{\prime}$ (Shuster and Wade 2003). 
Several authors have questioned the consistency of $I, I_{\mathrm{s}}$, and $\beta_{\text {ss }}$ in relation to realized sexual selection (e.g., Kokko et al. 1999; Jones et al. 2004; Klug et al. 2010). A major difficulty arises because under certain circumstances $I, I_{s}$, and $\beta_{\mathrm{ss}}$ may differ from realized sexual selection quantified using $\beta^{\prime}$. For example, in situations where differences in variance in lifetime mating success do not exist but differences in environmental variance do (e.g., in longevity and mate-encounter rate), differences may exist between populations in variance measures but not in $\beta^{\prime}$ (Hubbell and Johnson 1987; Grafen 1988). This is because variance measures include random variation in reproductive success that is not caused by sexual selection (Koenig and Albano 1986), whereas estimates of selection based on $\beta^{\prime}$ are less sensitive to random variation in fitness (Sutherland 1985; Koenig and Albano 1986; Hubbell and Johnson 1987; Grafen 1988; Klug et al. 2010). Variance measures may therefore provide little information regarding sexual selection in progress (realized sexual selection). In contrast, other studies have suggested that $I, I_{\mathrm{s}}$, and $\beta_{\mathrm{ss}}$ are valid means of estimating the strength and direction of sexual selection because they are closely tied to variation in mating success, a fundamental process of sexual selection (Shuster and Wade 2003; Jones et al. 2004). However, to date few studies have investigated the consistency of these proposed indices of sexual selection (Jones et al. 2000, 2004; Mills et al. 2007).

A lack of consensus also exists regarding the pools of individuals that should be used to measure sexual selection (Emlen and Oring 1977; Clutton-Brock and Vincent 1991; Clutton-Brock and Parker 1992; see also Klug et al. 2010). For example, male sand gobies (Pomatoschistus minutus) compete among each other for nesting sites, after which females select males occupying suitable sites (Kvarnemo 1996; Kvarnemo and Ahnesjö 1996). Female mate choice has been clearly attributed to sexual selection, but male competition for nesting sites may be part of sexual selection or resource competition and thus potentially influenced by natural selection (Ahnesjö et al. 2001). Consequently, estimates of realized sexual selection may be based on all competing males or only on males subject to female mate choice. A similar difficulty arises in species in which males attain sexual maturity at different ages. A nonreproductive male may be excluded from mating because of sexual selection (Vanpe et al. 2008) or because he is not ready to reproduce, which may be due to natural selection (e.g., Clutton-Brock and Parker 1992). These different potential sets of individuals used for the quantification of realized sexual selection are hereafter referred to as different "pools of individuals."

Another source of confusion comes from the fact that sexual selection includes premating processes, such as intrasexual competition for mates and mate choice, and postmating processes, such as sperm competition, cryptic female choice, and differential postfertilization and posthatching investment (Andersson 1994; Eberhard 1996). Proponents of sexual selection indices such as $I, I_{s}$, and $\beta_{\mathrm{ss}}$ argue that sexual selection arises primarily through selection on access to mates, in line with the original ideas of Darwin and Bateman (e.g., Shuster and Wade 2003; Jones et al. 2004). However, sexual selection during mating (e.g., differential sperm investment) and postmating sexual selection (e.g., cryptic female choice and differential attractiveness) can also generate substantial variation in the fitness of parents and offspring (Eberhard 1996; Head et al. 2005). Thus, use of different fitness proxies — such as access to mates (i.e., fertilization success), number of mates (number of mates with which at least one fertilized egg was shared), number of fertilized eggs, and number of offspring - may lead to differences in estimates of sexual selection, depending on when sexual selection occurs (e.g., Fitze and Le Galliard 2008). Unfortunately, previous comparative studies of indices of sexual selection used one (number of mates) or two (number of mates and number of offspring) fitness proxies to quantify sexual selection (Jones et al. 2000, 2004; Mills et al. 2007) and thus may have missed important selective events, such as differential investment into offspring, selective abortion, and selective killing of offspring (e.g., Lyon et al. 1994).

In summary, debate exists over the consistency of different fitness proxies, pools of individuals, and sexual selection indices. Inconsistencies could potentially generate substantial biases within and among studies, compromising their interpretation and hindering meta-analyses (Vanpe et al. 2008; Klug et al. 2010). Using the common lizard (Lacerta vivipara), we investigated whether different indices, pools of individuals, and fitness proxies qualitatively and quantitatively affect the conclusions of experimental adult sex ratio (ASR) manipulation, which is tightly linked to sexual selection. The common lizard is a nonterritorial species with a polygynandrous mating system in which sexual selection is determined by intrasexual competition among males (dominance of big males), female mate preferences for larger males, and male mate preferences for attractive females (Fitze and Le Galliard 2008; Fitze et al. 2010; P. S. Fitze, unpublished data). Furthermore, males may compromise female reproductive success through sexual harassment, particularly in situations of male-biased sex ratios (Le Galliard et al. 2005b, 2008). We previously showed that access to mates and male-induced female mortality are important determinants of sexual selection in common lizards (Fitze and Le Galliard 2008). We measured the intensity of sexual selection acting on males and females by calculating $I, I_{\mathrm{s}}$, $\beta_{\text {ss }}$, and $\beta^{\prime}$ with respect to body size (one of the most important determinants of male and female fitness), com- 
petitive ability, and survival in common lizards (Bauwens and Verheyen 1987; Le Galliard et al. 2004, 2005a; Fitze and Le Galliard 2008). We used three different pools of individuals — namely, restricted pools (including only lizards that reproduced), operational pools (including all lizards present during the mating period), and inclusive pools (including all lizards introduced into the experimental populations 8 months before reproduction). To understand the importance of the different components of sexual selection, we used premating and postmating fitness proxies-namely, access to mates, number of genetic mates, number of fertilized eggs, number of hatchlings, and number of yearlings - and we statistically analyzed differences among indices and pools.

If sexual selection was based on access to mates, as has been suggested for male common lizards (Fitze and Le Galliard 2008), we predicted that there would be significant differences among ASR treatments for all fitness proxies of inclusive and operational pools but not for restricted pools, because the ASR is a primary determinant of mating competition among males. Given that male-induced mating mortality and female mate choice are important for female fitness (Fitze and Le Galliard 2008), we also expected that there would be quantitative differences between estimates of sexual selection based on different pools of individuals. For females, we predicted that there would be differences between inclusive and operational pools due to mating mortality induced by ASR treatment but no differences between operational and restricted pools. In contrast, if postmating sexual selection was more important than premating sexual selection, we predicted that there would be no significant ASR effects on access to mates and number of mates but that there would be significant differences in the number of fertilized eggs and the number of offspring, independent of the pools used. We also expected to find quantitative differences between pools of individuals for $I$ and $I_{s}$, given that different pools are based on different samples and different selection processes, but not for $\beta^{\prime}$, for which differences in sample sizes play a minor role and selection may be more consistent. Finally, we predicted that there would be significant positive correlations among indices of sexual selection, pools of individuals, and fitness proxies and no qualitative differences among indices of sexual selection for any fitness proxies or pools of individuals if indeed all indices measured realized sexual selection consistently.

\section{Material and Methods}

\section{Species Description}

The common lizard (Lacerta vivipara) is a small ovoviviparous and polygynandrous lizard that lives in peat bogs and moist heathlands across Eurasia (Massot et al. 1992). All age and sex classes occupy nonexclusive territories, which allows manipulative study in field enclosures (Le Galliard et al. 2005b). Mating occurs immediately after the emergence of females from hibernation, approximately $3-$ 5 weeks after the emergence of males. In natural and seminatural populations, females mate with three different males on average, and males sire offspring with up to 14 different females (Laloi et al. 2004; Fitze et al. 2005; Richard et al. 2005). After mating, females incubate the eggs for approximately 7-9 weeks until parturition and thereafter no longer care for their offspring (Fitze et al. 2005). In our study area, females lay one clutch per year.

\section{Experimental Manipulation of the ASR}

In July 2002, 12 experimental lizard populations were established in outdoor enclosures $(10 \mathrm{~m} \times 10 \mathrm{~m})$ located at the Centre de Recherche en Ecologie Expérimentale et Prédictive (CEREEP)-Ecotron IleDeFrance (St-Pierre-lèsNemours, France). Each enclosure contained a patch of natural vegetation, two water ponds (which provided natural food and water), and four stone piles used as basking sites and shelters. Plastic walls prevented lizards from escaping from the enclosures. Eighteen adults (more than 1 year old), 12 yearlings ( 1 year old), and 42-45 newborns of known sex were released into each enclosure at the start of the experiment. In six of the 12 populations, we biased the ASR (defined as the number of adult males per the number of adult individuals) toward males (male-biased [MB] treatment); in the other six populations, we biased the ASR toward females (female-biased [FB] treatment). The yearling and newborn sex ratio was 0.5 . Fourteen adult males and four adult females were released into MB populations (ASR $=0.78$ ), and four adult males and 14 adult females were released into FB populations (ASR $=0.22$ ). The FB sex ratio corresponded to the average ASR, and the MB sex ratio corresponded to the uppermost ASRs $($ ASR $=0.80)$ of similarly sized patches in a natural common lizard population (average ASR $=0.22 \pm 0.21 \mathrm{SD}$ in 22 patches monitored over 13 years [data provided by M. Massot]; Le Galliard et al. 2005b).

\section{Assessment of Reproductive Characteristics}

During gestation, 1 month after the beginning of the mating season (late May 2003), all surviving lizards were captured. At this time, population sex ratios (SRs) still differed between treatments ( $\mathrm{MB}$ populations: $23.7 \pm 3.2 \mathrm{SE}$ males, $5.7 \pm 0.8$ SE females, SR $=0.80 \pm 0.03 \mathrm{SE}$; FB populations: $15.8 \pm 2.5 \mathrm{SE}$ males, $20.7 \pm 2.1 \mathrm{SE}$ females, $\left.\mathrm{SR}=0.40 \pm 0.03 \mathrm{SE} ; N=12, F_{1,10}=113.24, P<.001\right)$. Lizards were not captured during the mating season be- 
cause this may affect mate choice, mate competition, and estimates of the intensity of sexual selection. The number of males observed at capture corresponded closely to the number of males present during mating in both treatments, since male survival was high in spring and not affected by ASR manipulation (Le Galliard et al. 2005b). After capture, the snout-vent length (SVL) of each lizard was measured to the nearest millimeter. Captured females were maintained in the laboratory in individual terrariums under standardized conditions and were fed moth larvae (Pyralis sp.) every fourth day (Le Galliard et al. 2003).

After parturition, we carefully searched for hatchlings and unhatched eggs. Two days after hatching, offspring were released into eight initially empty enclosures similar to those used for the ASR experiment. We released the same number of families into each population, and the proportion of offspring originating from $\mathrm{MB}$ and $\mathrm{FB}$ populations was similar for each population (Fitze and Le Galliard 2008; Le Galliard et al. 2008). All surviving offspring were recaptured approximately 11 months later, in June 2004. This common-garden protocol allowed quantification of potential delayed effects of ASR manipulation on offspring viability independently of the direct effects of ASR.

\section{Genetic Protocol and Assessment of Paternity}

We collected a tissue sample from each individual released in $2002(N=879)$ and from each hatchling $(N=687)$ and unhatched egg $(N=66)$ in 2003. All tissues were stored in $60 \%$ ethanol, and DNA was extracted from each sample by means of a Perfect gDNA Blood Mini Isolation kit for animal blood (Eppendorf). We used five or six highly polymorphic microsatellite DNA loci (Lv-3-19, Lv4-72, Lv-4- $\alpha$, Lv-4-X, Lv-4-115, and Lv-2-145; Boudjemadi et al. 1999) to identify fathers. The methods used for DNA extraction, polymerase chain reaction, and determination of allelic size have been described elsewhere (Laloi et al. 2004). DNA could not be extracted for seven unhatched eggs from four different mothers in FB populations and for five unhatched eggs from three different mothers in $\mathrm{MB}$ populations. We conducted assignment tests using Cervus 2.0 (Marshall et al. 1998) to identify the genetic father for each hatchling and unhatched egg (Fitze et al. 2005).

\section{Measurements of Sexual Selection}

We used three different methods to quantify sexual selection in each experimental population, hereafter referred to as "indices of sexual selection." First, we calculated the opportunity of sexual selection $\left(I_{s}\right)$, using access to mates (regardless of whether an individual produced fertilized eggs) and number of mates (number of mates with whom at least one fertilized egg was shared) as fitness proxies. We calculated the opportunity of selection (I) for the following fitness proxies: number of eggs, number of hatchlings, and number of yearlings (Shuster and Wade 2003; Wade and Shuster 2004). Given that $I_{s}$ and $I$ differ in the fitness proxy used but not in the mathematical formula, we hereafter use only I. Second, we calculated the Bateman gradient, which characterizes the relationship between reproductive success and mating success (number of genetic mates) using a least squares regression (Arnold and Duvall 1994). Third, we calculated the standardized selection gradient $\left(\beta^{\prime}\right)$ using a regression with standardized fitness measurement as the dependent variable and standardized SVL as a covariate (Lande and Arnold 1983). We calculated $\beta^{\prime}$ for SVL because body size is a sexually selected trait that strongly determines the reproductive success of male and female common lizards (Fitze et al. 2008, 2010; Fitze and Le Galliard 2008). For each index, we used three alternative pools of individuals. Inclusive pools included all lizards introduced into the experimental populations at the start of the experiment, and SVL at release was used to calculate $\beta^{\prime}$. Operational pools included all lizards that survived hibernation, and SVL after the mating period was used to calculate $\beta^{\prime}$. All surviving females laid eggs, and $95 \%$ of the surviving males had an SVL, for which egg fertilization was confirmed in this experiment. This shows that most surviving lizards were ready to mate and that for only a few individuals was readiness not certain. Restricted pools included only lizards that reproduced successfully-that is, that fathered at least one fertilized egg. For all indices and pools, we also used fitness proxies that are typically associated with different mechanisms of sexual selection (Andersson 1994; Eberhard 1996), including access to mates, number of mates, number of eggs, number of hatchlings, and number of yearlings. For males, fitness estimates were determined using paternity analyses.

\section{Statistical Analyses}

Table A1 in the online edition of the American Naturalist shows the sample sizes used for the calculation of each of the indices of sexual selection, pools of individuals, and fitness proxies. To investigate differences between ASR treatments, we calculated the three indices of sexual selection for all animals that were in direct competition and thus for each of the independent populations (enclosure). Data files have been deposited at Dryad (http://dx.doi.org/ 10.5061/dryad.8872). The three indices of sexual selection were also calculated for each combination of fitness proxies and each pool of individuals. We used independent estimates of sexual selection for each population because sexual selection relies on reproductive skew among competing 
and interacting individuals and because sexual selection indices are properties of populations rather than individuals (Andersson 1994; Jones et al. 2004). Sample size was $N=12$ populations except when the number of yearlings was used as a fitness proxy because in some populations too few lizards produced yearlings to allow calculation of $\beta^{\prime}, I$, or $\beta_{\mathrm{ss}}$. For males, the estimates of $\beta_{\mathrm{ss}}$ for restricted pools of individuals were based on smaller sample sizes because in two populations all reproducing males had the same number of mates. To test for differences between pools, we used repeated-measures ANOVA with inclusive, operational, and restricted pools as repeats (within-subject factor) and ASR treatment as a between-subject factor. To test for differences between fitness proxies, we used repeated-measures ANOVA with fitness proxies as repeats and ASR treatment as a factor. To determine whether different indices of sexual selection identify the same patterns of sexual selection, we conducted Spearman rank correlations (table A2 in the online edition of the American Naturalist). The assumptions of the statistical models were verified in all cases (Quinn and Keough 2002), and transformations were applied where necessary. For all tests we reported two-tailed probabilities with a significance level of .05. Where necessary, we applied sequential Bonferroni corrections to adjust $P$ values for the increased probability of achieving statistical significance from multiple tests. Results are reported as means $\pm 1 \mathrm{SE}$.

\section{Results}

\section{Sexual Selection on Males}

Comparison of Indices of Sexual Selection. Estimates of the opportunity of selection (I) were higher in MB populations (fig. $1 A$ ). In contrast, estimates of the standardized selection gradient $\left(\beta^{\prime}\right)$ for inclusive and operational pools indicated that sexual selection on male body size was stronger in FB populations (fig. 1A). The direction of the ASR treatment effect on sexual selection, measured over all pools of individuals and all fitness proxies (fig. 1A), differed significantly between estimates of $I$ and estimates of $\beta^{\prime}$ (McNemar test, $\left.N=14, N_{\text {changes }}=12, P<.001\right)$. Estimates of $I$ were significantly greater than estimates of $\beta^{\prime}$ in inclusive and operational analyses (Wilcoxon signedrank test for paired samples, all $P<.05$; fig. $1 A$ ) and in all but one restricted analysis (number of females). Estimates of the Bateman gradient $\left(\beta_{\mathrm{ss}}\right)$ were steeper in $\mathrm{FB}$ than in MB populations (fig. $1 A$ ). The direction of the ASR treatment effect did not differ between estimates of $\beta_{\mathrm{ss}}$ and estimates of $\beta^{\prime}$ (McNemar test, $N=8$, $N_{\text {changes }}=2, P=.289$ ) but differed significantly between estimates of $\beta_{\mathrm{ss}}$ and estimates of $I$ (McNemar test, $N=$ $\left.8, N_{\text {changes }}=6, P=.025\right)$. Estimates of $\beta_{\mathrm{ss}}$ were greater than estimates of $\beta^{\prime}$ for all pools and fitness proxies (Wilcoxon signed-rank test for paired sample, all $P<.05$; fig. $1 A$ ), less than estimates of $I$ in inclusive and operational pools (all $P<.05$; fig. $1 A$ ), and greater than estimates of $I$ in one measure using restricted pools (number of eggs: $P=.002$; fig. $1 A$ ). Only one significant positive and three significant negative correlations were detected between indices of sexual selection (table A2). All other correlations were not significant, indicating that different indices of sexual selection do not capture the same information.

Comparison of Pools of Individuals. Repeated-measures ANOVAs revealed significant differences in estimates of $I$ between pools and significant interactions between pools and ASR treatment, with the exception of access to mates (table 1, pt. A). Post hoc tests between pairs of pools showed significant differences between inclusive and operational pools for all fitness proxies (all $F_{1,10} \geq 20.901$, $P \leq .01)$ and no significant interactions between ASR treatment and pools (all $F_{1,10} \leq 1.659, P \geq .227$ ). Significant differences were also observed between restricted pools, on the one hand, and operational or inclusive pools, on the other hand, for all fitness proxies $\left(F_{1,8} \geq 36.654, P \leq\right.$ .001). The interaction between ASR treatment and pool pairs was significant in all but one case (number of mates, inclusive vs. restricted pools, nonsignificant interaction; all other interactions: $\left.F_{1,10} \geq 5.772, P \leq 0.037\right)$.

Repeated-measures ANOVAs revealed significant differences in estimates of $\beta_{\mathrm{ss}}$ between pools and a significant interaction between pools and ASR treatment in the number of hatchlings but not in other fitness proxies (table 1, pt. B). Post hoc tests showed significantly greater estimates of $\beta_{\mathrm{ss}}$ in restricted pools than in inclusive and operational pools (all $F_{1,8} \geq 33.651, P<.001$ ) and significantly greater estimates of $\beta_{\mathrm{ss}}$ for operational pools than for inclusive pools $\left(F_{1,10}=8.970, P=.014\right)$. A larger ASR treatment effect was observed in operational pools than in inclusive pools $\left(F_{1,10}=5.907, P=.035\right)$, but no significant differences in the ASR treatment effect were detected between the other pools $(P>.27)$. Repeated-measures ANOVAs revealed no significant differences in estimates of $\beta^{\prime}$ between pools and no significant interactions between pools and ASR treatment (table 1, pt. C).

Comparison of Fitness Proxies. Estimates of $I$ tended to differ between fitness proxies for inclusive pools and differed significantly for restricted pools, whereas no significant interactions between ASR treatment and fitness proxies were found (table 1, pt. A). Post hoc tests showed that estimates of $I$ significantly differed between the number of hatchlings and the other fitness proxies (all $F_{1,11} \geq$ $10.809, P \leq .042$ ), although no other differences were observed. Estimates of $\beta_{\mathrm{ss}}$ differed among fitness proxies in 
inclusive and operational pools, and significant interactions were observed between ASR treatment and fitness proxies (table 1, pt. B). Post hoc tests showed significant differences among all pools (all $F_{1,8} \geq 17.95, P<.002$ ). Estimates of $\beta_{\text {ss }}$ decreased from number of eggs to number of hatchlings and number of yearlings. Furthermore, the ASR treatment effect was larger when using the number of eggs or the number of hatchlings than when using the number of yearlings (all $F_{1,8} \geq 18.84, P<.008$ ). There were no significant differences in estimates of $\beta^{\prime}$ between fitness proxies in any of the pools and no significant interactions between ASR treatment and fitness proxies (table 1, pt. C).

\section{Sexual Selection on Females}

Comparison of Indices of Sexual Selection. Estimates of I were larger in $\mathrm{MB}$ than in $\mathrm{FB}$ populations for all pools (fig. 1B). In contrast, estimates of $\beta^{\prime}$ were steeper in $\mathrm{FB}$ than in $\mathrm{MB}$ populations for inclusive pools and were steeper in $\mathrm{MB}$ populations for operational and restricted pools (fig. 1B). The direction of the ASR treatment effect on sexual selection significantly differed between the two indices when using inclusive pools (McNemar test, $N=$ $\left.5, N_{\text {changes }}=5, P=.025\right)$, although no differences existed when using operational and restricted pools $(N=5$, $N_{\text {changes }}=0, P=1$; fig. $1 B$ ). Estimates of $I$ were greater than estimates of $\beta^{\prime}$ in inclusive and operational pools (Wilcoxon signed-rank test for paired samples, all $P<$ .05; fig. 1A) but not in restricted analyses (all $P \geq .2$ ). For inclusive and operational pools, estimates of $\beta_{\text {ss }}$ were greater in $\mathrm{FB}$ than in $\mathrm{MB}$ populations (fig. $1 B$ ). The direction of the ASR treatment effect significantly differed between estimates of $\beta_{\mathrm{ss}}$ and estimates of $I$ (McNemar test, $N=8, N_{\text {changes }}=6, P=.014$ ) but did not differ between estimates of $\beta_{\mathrm{ss}}$ and estimates of $\beta^{\prime}$ (McNemar test, $\left.N=8, N_{\text {changes }}=3, P=.248\right)$. Estimates of $\beta^{\prime}$ were smaller than estimates of $\beta_{\text {ss }}$ in inclusive analyses and in one analysis using operational pools (number of eggs: Wilcoxon signed-rank test for paired samples, all $P<.05$; fig. $1 B)$ but not in restricted analyses $(P \geq .2)$. Estimates of $\beta_{\text {ss }}$ were smaller than estimates of $I$ in two inclusive pools (number of hatchlings and number of yearlings) and in all operational pools (Wilcoxon signed-rank test for paired samples, all $P<.05$; fig. $1 A$ ) but not in restricted pools $(P>.1)$. Two significant positive and four significant negative correlations were observed between indices of sexual selection (table A2), but most of the correlations were not significant $(N=26)$, showing that different indices do not capture the same information.

Comparison of Pools of Individuals. Repeated-measures ANOVAs revealed significant differences in estimates of $I$ between pools and significant interactions between pools and ASR treatment (table 1, pt. A). Post hoc tests showed significantly greater estimates of $I$ in inclusive pools than in operational or restricted pools for all fitness proxies (all $\left.F_{1,10} \geq 173.535, P<.001\right)$. Furthermore, the ASR treatment effect was significantly greater in inclusive pools than in operational or restricted pools (all $F_{1,10} \geq 16.038, P \leq$ .010). Differences existed between operational and restricted pools for all fitness proxies $\left(F_{1,10} \geq 19.634, P \leq\right.$ .005). The ASR treatment effect did not significantly differ between operational and restricted pools (all $F_{1,10} \leq$ 1.572, $P \geq .239)$.

Estimates of $\beta_{\mathrm{ss}}$ differed significantly between pools, and significant interactions between pools and ASR treatment were found for all fitness proxies with the exception of the number of yearlings (table 1, pt. B). Post hoc tests revealed significantly greater estimates of $\beta_{\mathrm{ss}}$ in inclusive and operational pools than in restricted pools (all $F_{1,10} \geq$ 5.523, $P \leq .041)$ and a larger ASR treatment effect in inclusive and operational pools than in restricted pools (all $\left.F_{1,10} \geq 7.215, P \leq .046\right)$. Repeated-measures ANOVAs revealed highly significant differences in estimates of $\beta^{\prime}$ between inclusive, operational, and restricted pools and significant interactions between pools and ASR treatment (table 1, pt. C). Post hoc tests indicated smaller estimates of $\beta^{\prime}$ for inclusive pools than for operational pools (all $P<.003$ ) and restricted pools (all $P<.05$, except for number of mates). The ASR treatment effect was larger in inclusive pools than in operational pools (all $P \leq .032$ ) and restricted pools (all $P \leq .030$ ) with the exception of the number of yearlings, where the ASR treatment effect was stronger in restricted pools $(P=.050)$. There were no significant differences between operational and restricted pools (all $P>.3$ ).

Comparison of Fitness Proxies. Repeated-measures ANOVAs revealed significant differences in estimates of $I$ between fitness proxies when using inclusive and operational pools. No significant differences were found when using restricted pools, and the interaction between ASR treatment and fitness proxies was significant only for inclusive pools (table 1, pt. A). Estimates of $I$ were significantly smaller when using access to mates as a fitness proxy compared with other fitness proxies, for both inclusive and operational pools (table A3 in the online edition of the American Naturalist). The significance and direction of the differences between the other fitness proxies are presented in table A3.

Significant differences in estimates of $\beta_{\text {ss }}$ were observed between fitness proxies for all pools, and a significant interaction between ASR treatment and fitness proxies existed for inclusive pools (table 1, pt. B). Post hoc tests on inclusive pools showed that estimates of $\beta_{\text {ss }}$ calculated using the number of yearlings were less steep than those 

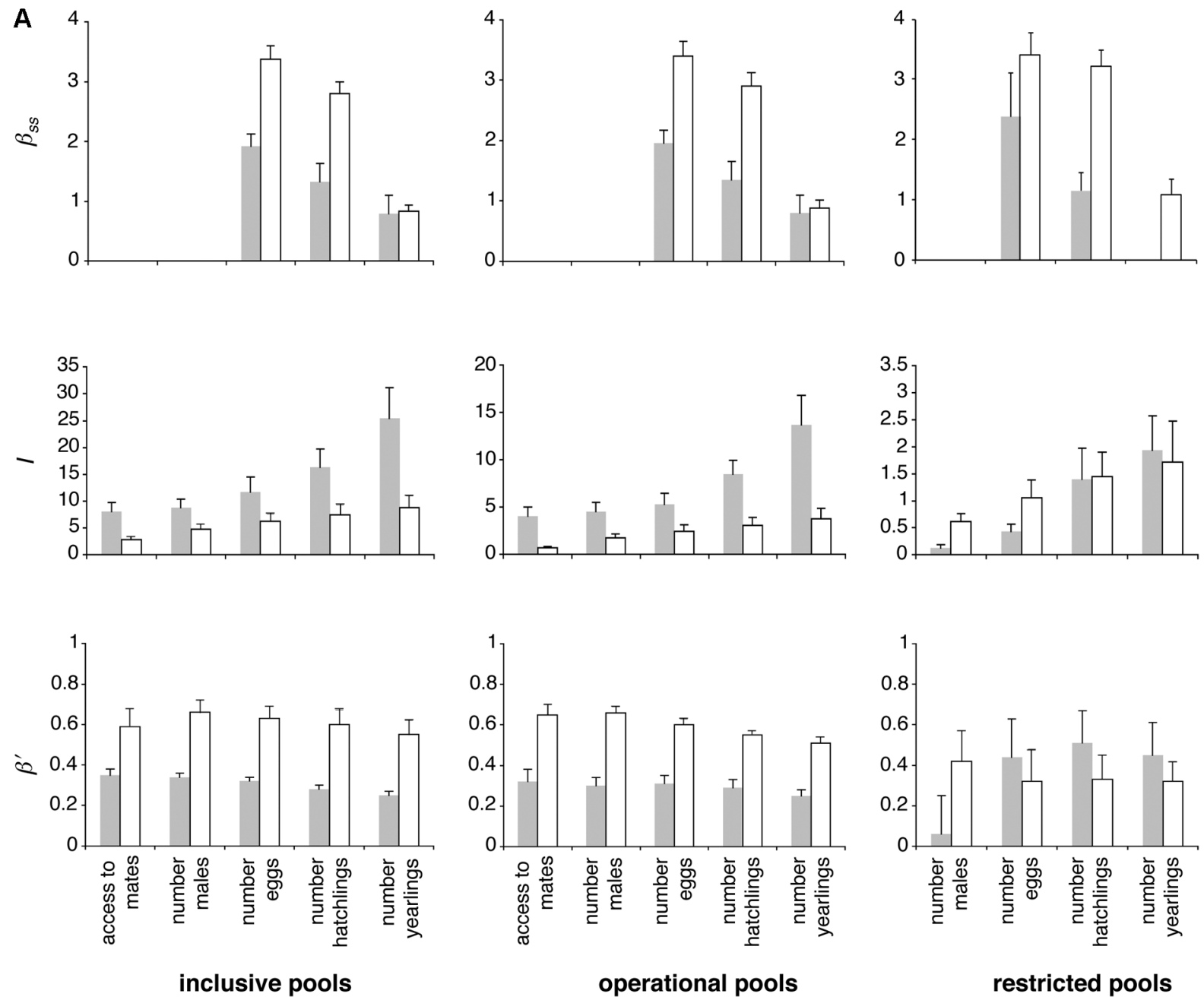

Figure 1: Effects of adult sex ratio treatment on sexual selection acting on $(A)$ male and $(B)$ female common lizards. Means and SE are based on six male-biased (gray bars) and six female-biased (white bars) populations and are given for each sex, for each of three indices of sexual selection (the standardized selection gradient $\left[\beta^{\prime}\right]$, the opportunity for selection $[I]$, and the Bateman gradient $\left[\beta_{\mathrm{ss}}\right]$ ), for each of three pools of individuals (inclusive, operational, and restricted pools), and for each of five fitness proxies. For estimates of $\beta^{\prime}$ and $\beta_{\mathrm{ss}}$, the $Y$-axis scale uses the same units for all three measures of sexual selection; this is not the case for estimates of $I$.

calculated using the number of eggs or the number of hatchlings $(P<.001)$. Repeated-measures ANOVAs revealed significant differences in estimates of $\beta^{\prime}$ between fitness proxies in two of the three pools. Two of three interactions between ASR treatment and fitness proxies were significant (table 1, pt. C). There were no common patterns in differences between fitness proxies, although for inclusive pools, using access to mates, estimates of $\beta^{\prime}$ were generally smaller than those for other fitness proxies (table A3).

\section{Discussion}

The results of our detailed analyses partly contradict those of earlier studies of fish, small mammals, newts, and seed bugs, which reported consistency between different indices of sexual selection (McLain 1992; Jones et al. 2000, 2004; Mills et al. 2007). McLain (1992) and Mills et al. (2007) reported that different indices of sexual selection provided comparable results, and Mills et al. (2007) found significant positive correlations among the different indices. McLain (1992), Jones et al. (2004), and Mills et al. (2007) also found that different fitness proxies provided qualitatively similar intensities of sexual selection, and Jones et al. (2000) reported similar intensities of sexual selection using different pools of individuals. In contrast, using a powerful and very precise experimental ASR manipulation with 12 independent lizard populations and more than 800 lizards, we found qualitative and quantitative differ- 

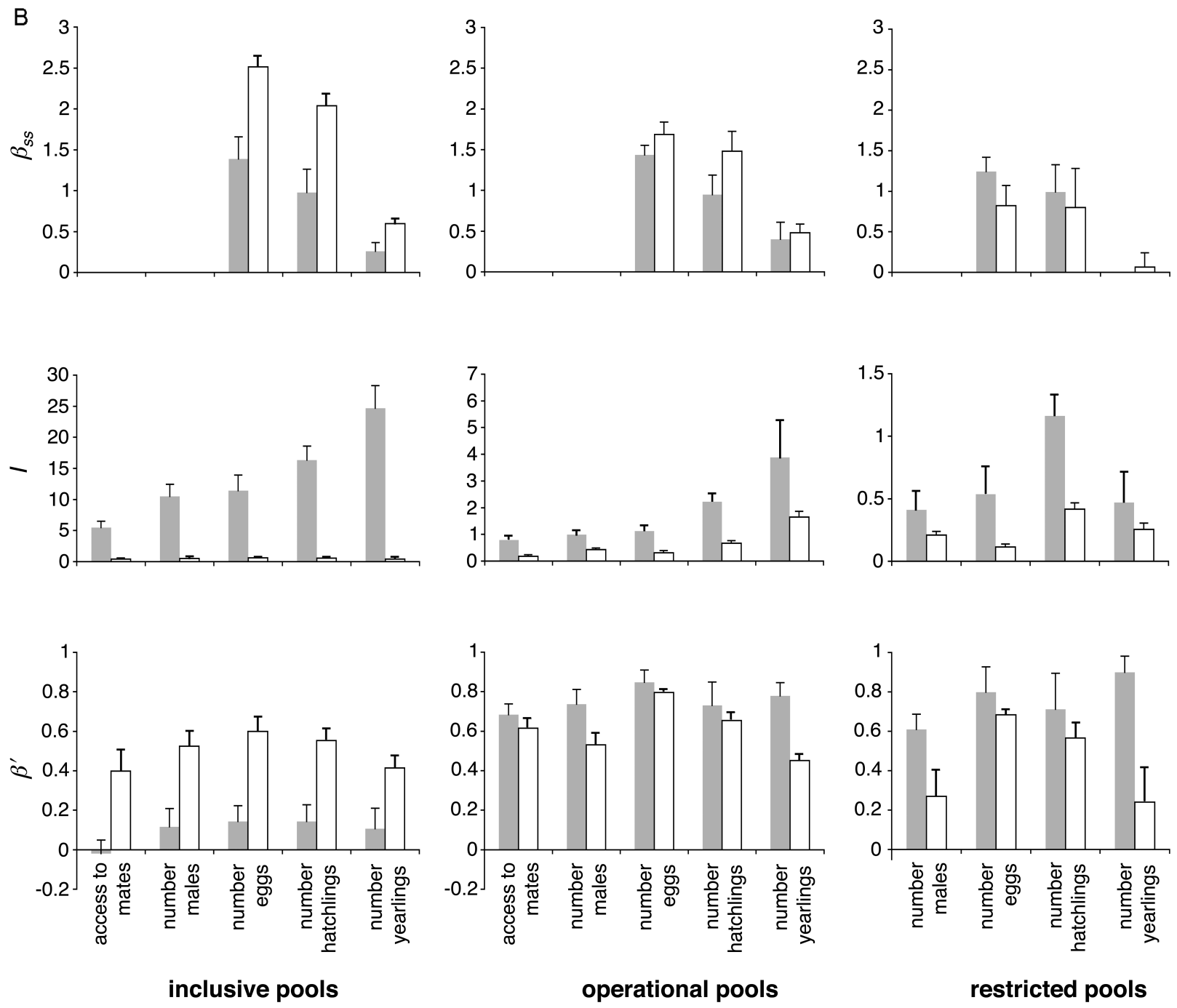

Figure 1: (Continued)

ences in the estimated intensity of sexual selection between three commonly used indices of sexual selection-namely, the opportunity of selection $(I)$, the Bateman gradient $\left(\beta_{\mathrm{ss}}\right)$, and the standardized selection gradient $\left(\beta^{\prime}\right)$-with respect to body size. We also found few positive correlations among the different indices and significant differences in the intensity and direction of sexual selection when using different fitness proxies and different pools of individuals.

In males, the estimated intensity of sexual selection was stronger in $\mathrm{MB}$ than in $\mathrm{FB}$ populations when using $I$, but it was stronger in $\mathrm{FB}$ populations when using $\beta_{\mathrm{ss}}$ and $\beta^{\prime}$. Furthermore, we observed quantitative differences and no significant positive correlations between estimates of $\beta_{\mathrm{ss}}$ and estimates of $\beta^{\prime}$. These results are in line with the findings of Westneat (2006) and Klug et al. (2010), who concluded that $I$ is not always consistent with $\beta^{\prime}$, quantified with respect to sexually dimorphic traits targeted by sexual selection. The ability of males to monopolize females was proposed to contribute to the differences between $I$ and $\beta^{\prime}$ in a recent model of sexual selection (Klug et al. 2010) and in a comparative analysis (Weir et al. 2011). The greater estimates of $I$ observed in males of MB populations may thus be the result of more intense sexual selection or increased random variation in fitness (e.g., Klug et al. 2010). The latter hypothesis seems particularly relevant to our findings. In $\mathrm{MB}$ populations, estimates of $\beta^{\prime}$ were smaller, relatively more males competed for access to females, and the number of fertilizable eggs per female and per population was smaller (Sutherland 1985; Westneat 
Table 1: Repeated-measures ANOVA with pools of individuals or fitness proxies as repeated measures (within-subject factor) and adult sex ratio (ASR) treatment as a factor

\begin{tabular}{|c|c|c|c|c|}
\hline \multirow{2}{*}{$\begin{array}{l}\text { Sex, within-subject factor, } \\
\text { factor analysis is based on }\end{array}$} & \multicolumn{2}{|c|}{ Within-subject effect } & \multicolumn{2}{|c|}{$\begin{array}{c}\text { ASR } \times \\
\text { within-subject effect }\end{array}$} \\
\hline & $F(\mathrm{df})$ & $P$ & $F(\mathrm{df})$ & $P$ \\
\hline & \multicolumn{4}{|c|}{ A. Sexual selection measured using estimates of $I$} \\
\hline \multicolumn{5}{|l|}{ Males: } \\
\hline \multicolumn{5}{|l|}{ Pools: ${ }^{\mathrm{a}}$} \\
\hline Access to mates & $22.563(1,10)$ & $<.001$ & $2.145(1,10)$ & .174 \\
\hline No. mates & $21.409(2,9)$ & $<.001$ & $4.342(2,9)$ & .048 \\
\hline No. eggs & $47.348(2,8)$ & $<.001$ & $5.610(2,8)$ & .030 \\
\hline No. hatchlings & $22.143(2,9)$ & $<.001$ & $8.501(2,9)$ & .008 \\
\hline No. yearlings & $24.580(2,7)$ & .001 & $9.443(2,7)$ & .010 \\
\hline \multicolumn{5}{|l|}{ Fitness proxies: ${ }^{\mathrm{b}}$} \\
\hline Inclusive pools & $4.451(4,5)$ & .067 & $1.977(4,5)$ & .236 \\
\hline Operational pools & $3.312(4,4)$ & .136 & $1.536(4,4)$ & .344 \\
\hline Restricted pools & $17.898(3,5)$ & .004 & $2.313(3,5)$ & .193 \\
\hline \multicolumn{5}{|l|}{ Females: } \\
\hline \multicolumn{5}{|l|}{ Pools: ${ }^{\mathrm{a}}$} \\
\hline Access to mates & $61.026(1,10)$ & $<.001$ & $8.622(1,10)$ & .015 \\
\hline No. mates & $416.699(2,9)$ & $<.001$ & $28.285(2,9)$ & $<.001$ \\
\hline No. eggs & $196.019(2,9)$ & $<.001$ & $4.293(2,9)$ & .049 \\
\hline No. hatchlings & $271.338(2,9)$ & $<.001$ & $29.620(2,9)$ & $<.001$ \\
\hline No. yearlings & $616.452(2,5)$ & $<.001$ & $83.216(2,5)$ & $<.001$ \\
\hline \multicolumn{5}{|l|}{ Fitness proxies: ${ }^{\mathrm{b}}$} \\
\hline Inclusive pools & $44.964(4,5)$ & $<.001$ & $5.826(4,5)$ & .040 \\
\hline Operational pools & $26.076(4,5)$ & .002 & $1.610(4,5)$ & .304 \\
\hline \multirow[t]{2}{*}{ Restricted pools } & $4.651(3,4)$ & .086 & $2.250(3,4)$ & .225 \\
\hline & \multicolumn{4}{|c|}{ B. Sexual selection measured using estimates of $\beta_{s \mathrm{~s}}$} \\
\hline \multicolumn{5}{|l|}{ Males: } \\
\hline \multicolumn{5}{|l|}{ Pools: ${ }^{\mathrm{a}}$} \\
\hline No. eggs & $.759(2,7)$ & .503 & $1.614(2,7)$ & .265 \\
\hline No. hatchlings & $27.874(2,7)$ & $<.001$ & $7.952(2,7)$ & .016 \\
\hline No. yearlings & $3.252(1,8)$ & .109 & $2.716(1,8)$ & .138 \\
\hline \multicolumn{5}{|l|}{ Fitness proxies: ${ }^{\mathrm{b}}$} \\
\hline Inclusive pools & $52.658(2,7)$ & $<.001$ & $8.894(2,7)$ & .012 \\
\hline Operational pools & $49.028(2,7)$ & $<.001$ & $8.692(2,7)$ & .013 \\
\hline Restricted pools & $4.022(1,8)$ & .080 & $2.184(1,8)$ & .178 \\
\hline \multicolumn{5}{|l|}{ Females: } \\
\hline \multicolumn{5}{|l|}{ Pools: ${ }^{\mathrm{a}}$} \\
\hline No. eggs & $10.279(2,9)$ & .005 & $5.684(2,9)$ & .025 \\
\hline No. hatchlings & $4.268(2,9)$ & .050 & $5.633(2,9)$ & .026 \\
\hline No. yearlings & $.016(1,10)$ & .901 & $1.321(1,10)$ & .277 \\
\hline \multicolumn{5}{|l|}{ Fitness proxies: ${ }^{\mathrm{b}}$} \\
\hline Inclusive pools & $64.849(2,9)$ & $<.001$ & $5.254(2,9)$ & .031 \\
\hline Operational pools & $35.346(2,9)$ & $<.001$ & $1.473(2,9)$ & .280 \\
\hline \multirow[t]{2}{*}{ Restricted pools } & $10.252(1,10)$ & .01 & $.207(1,10)$ & .659 \\
\hline & C. Sexual selec & n meas & ed using estima & s of $\beta^{\prime}$ \\
\hline Males: & & & & \\
\hline Pools: ${ }^{\mathrm{a}}$ & & & & \\
\hline Access to mates & $.032(1,10)$ & .862 & $.971(2,10)$ & .348 \\
\hline No. mates & $2.050(2,8)$ & .191 & $.032(2,8)$ & .969 \\
\hline No. eggs & $.198(2,9)$ & .824 & $1.108(2,9)$ & .381 \\
\hline No. hatchlings & $.115(2,9)$ & .893 & $2.255(2,9)$ & .161 \\
\hline No. yearlings & $.073(2,7)$ & .930 & $2.529(2,7)$ & .149 \\
\hline Fitness proxies: & & & & \\
\hline Inclusive pools & $2.779(4,5)$ & .146 & $.760(4,5)$ & .593 \\
\hline Operational pools & $2.117(4,5)$ & .216 & $1.259(4,5)$ & .395 \\
\hline Restricted pools & $.765(3,5)$ & .561 & $1.727(3,5)$ & .277 \\
\hline
\end{tabular}


Table 1 (Continued)

\begin{tabular}{|c|c|c|c|c|}
\hline \multirow{2}{*}{$\begin{array}{l}\text { Sex, within-subject factor, } \\
\text { factor analysis is based on }\end{array}$} & \multicolumn{2}{|c|}{ Within-subject effect } & \multicolumn{2}{|c|}{$\begin{array}{c}\text { ASR } \times \\
\text { within-subject effect }\end{array}$} \\
\hline & $F(\mathrm{df})$ & $P$ & $F(\mathrm{df})$ & $P$ \\
\hline & \multicolumn{4}{|c|}{ C. Sexual selection measured using estimates of $\beta^{\prime}$} \\
\hline \multicolumn{5}{|l|}{ Females: } \\
\hline \multicolumn{5}{|l|}{ Pools: ${ }^{\mathrm{a}}$} \\
\hline Access to mates & $34.320(1,10)$ & $<.001$ & $9.595(1,10)$ & .011 \\
\hline No. mates & $22.057(2,9)$ & $<.001$ & $12.520(2,9)$ & .003 \\
\hline No. eggs & $128.993(2,9)$ & $<.001$ & $17.878(2,9)$ & .001 \\
\hline No. hatchlings & $36.731(2,9)$ & $<.001$ & $8.375(2,9)$ & .009 \\
\hline No. yearlings & $38.130(2,5)$ & $<.001$ & $35.820(2,5)$ & .001 \\
\hline \multicolumn{5}{|l|}{ Fitness proxies: ${ }^{\mathrm{b}}$} \\
\hline Inclusive pools & $31.521(4,5)$ & .001 & $25.618(4,5)$ & .002 \\
\hline Operational pools & $19.688(4,5)$ & .003 & $12.094(4,5)$ & .009 \\
\hline Restricted pools & $2.393(3,4)$ & .209 & $1.696(3,4)$ & .305 \\
\hline
\end{tabular}

2006; Klug et al. 2010). These factors may have led to scramble competition for access to mates (Weir et al. 2011), resulting in more male aggression toward females, as evidenced by an increased number of mating scars (Fitze et al. 2005; Le Galliard et al. 2005b). More intense sexual selection cannot explain the larger estimates of $I$ in $\mathrm{MB}$ populations because females of $\mathrm{MB}$ populations exerted less precopulatory sexual selection on males (Fitze and Le Galliard 2008), had higher costs of mating (increased female mortality [Le Galliard et al. 2005b] and lower reproductive investment [Le Galliard et al. 2008]), and suffered from increased male aggression (Le Galliard et al. $2005 b$ ). Consequently, proportionally more smaller males were able to mate in $\mathrm{MB}$ than in $\mathrm{FB}$ populations, resulting in weaker sexual selection on male body size (Fitze et al. 2005; Fitze and Le Galliard 2008).

In males, estimates of $I$ significantly differed among all pools of individuals and were largest for inclusive pools and smallest for restricted pools of individuals. In both males and females, estimates of $\beta_{\mathrm{ss}}$ were significantly smaller in restricted pools of individuals than in inclusive and operational pools. The decreases in estimates of $I$ and $\beta_{\mathrm{ss}}$ from inclusive to operational and restrictive pools occurred in parallel with decreasing sample sizes and shifts from premating to postmating selection. In males, estimates of $\beta^{\prime}$ provided relatively similar qualitative and quantitative results that were independent of the pool of individuals used (i.e., there were no significant differences between pools and no significant interaction between pools and ASR treatment; table 1, pt. C). In females, the intensity of sexual selection measured using estimates of $\beta^{\prime}$ in inclusive pools was the opposite of that found in operational and restricted pools. The stronger sexual selection in FB populations with inclusive pools can be explained by dif- ferences between ASR treatments in size-dependent female mortality during the mating period due to male sexual harassment (Le Galliard et al. 2005b). In FB populations, body size at the start of the experiment was larger in females that survived until egg laying (SVL $=48.30 \pm$ $1.60 \mathrm{~mm})$ than in females that did not $(\mathrm{SVL}=33.89 \pm$ $\left.1.54 \mathrm{~mm} ; F_{1,244}=42.33, P<.001\right)$. In MB populations, no differences were observed in body size between surviving and nonsurviving females at the start of the experiment (survivors: $33.82 \pm 2.69 \mathrm{~mm}$; nonsurvivors: $33.69 \pm$ $1.25 \mathrm{~mm} ; F_{1,188}=0.002, P=.963$; ASR treatment $\times$ survival interaction: $\left.F_{1,437}=14.03, P<.001\right)$. In contrast, sexual selection measured using estimates of $\beta^{\prime}$ did not differ between operational and restricted pools and was stronger in $\mathrm{MB}$ than in FB populations. The stronger sexual selection in $\mathrm{MB}$ populations was the result of increased multiple mating of large females in MB populations and associated fitness advantages due to increased ovulation (Fitze and Le Galliard 2008). Thus, in females differences between inclusive pools and other pools resulted from different selective events - namely, sex ratio-dependent survival selection and sex ratio-dependent reproductive advantages of larger body size-whereas differences in estimates of $I$ and $\beta_{\text {ss }}$ between pools were most likely due to a combination of differences in sample size and different selective events (Fitze and Le Galliard 2008).

The ASR treatment effect measured using estimates of $I$ and $\beta_{\text {ss }}$ significantly differed among fitness proxies in both males and females, contradicting the expectation that all indices, pools, and fitness proxies measure realized sexual selection with the same accuracy. Estimates of $\beta^{\prime}$ provided relatively constant estimates of ASR effects among fitness proxies for males but not for females (table 1, pt. $\mathrm{C}$; table A3), in which no clear patterns were apparent for 
differences among fitness proxies with respect to the ASR treatment effect. These results indicate that estimates of $\beta^{\prime}$ were consistent among pools in both males and females as long as the same selective mechanisms were quantified but were not consistent among different selective mechanisms.

We previously reported that sexual selection with respect to body size in male common lizards primarily acted on access to mates, whereas selection on female body size was mainly due to premating mortality and access to mates (Fitze and Le Galliard 2008). Consequently, the use of inclusive and operational pools led to different conclusions in females but similar conclusions in males. In this study, no differences between operational and restricted pools were observed, although such differences may exist in species with cryptic female mate choice due to sperm selection, selective abortion, and selective killing of offspring (e.g., Lyon et al. 1994). It is important to realize that the comparison of different pools does not constitute the best method for identifying the traits on which sexual selection acts, since measures using different pools are highly intercorrelated and assigning offspring fitness to parental fitness may be problematic because it can produce incorrect estimates of selection (Wolf and Wade 2001). Alternative methods for pinpointing selective events and causal pathways involve fitness decomposition (e.g., Fitze and Le Galliard 2008) and cautious consideration of selection mechanisms (Wolf and Wade 2001). Previous fitness decomposition based on operational pools of individuals using estimates of $\beta^{\prime}$ led to conclusions consistent with those of this study, finding that sexual selection mainly acts on female mortality during the mating period and on access to mates both in males and in females but not on the other multiplicative fitness proxies (Fitze and Le Galliard 2008).

The operational sex ratio, which we experimentally altered using an ASR manipulation, is in many species an important predictor of the intensity of sexual selection. In our experiment, ASR accounted for $31.5 \%$ of the variance in reproductive success (table A4 and fig. A1 in the online edition of the American Naturalist), whereas differences in the ASR treatment effect among populations with the same ASR treatment accounted for only $1 \%$ of the variance. Therefore, differences observed among indices of sexual selection and differences among fitness proxies and pools of individuals when using the same index indicate inconsistencies in measurements of sexual selection rather than differences in the ASR treatment effect among population replicates of the same ASR. These inconsistencies were sufficiently great to produce opposing patterns of sexual selection. This is an important result given that ASR treatment accounted for a significant amount of the variance in reproductive success (31.5\%). In general, estimates of $\beta^{\prime}$ provided qualitatively and quantitatively more consis- tent measurements of the intensity of realized sexual selection among different fitness proxies and different pools of individuals than did estimates of $I$ and $\beta_{\mathrm{ss}}$. Moreover, results based on estimates of $\beta^{\prime}$ better matched previous findings and patterns predicted by the mating system. Although estimates of $\beta^{\prime}$ generally performed better, it is not possible to evaluate the degree to which realized sexual selection acting on body size reflects sexual selection on other traits. Differences in the intensity of sexual selection estimated using different indices of sexual selection and the general lack of significant correlations among indices demonstrate that the use of different indices often leads to divergent estimates of realized sexual selection. Estimates of $\beta^{\prime}$ unraveled different selective mechanisms acting on female body size but were consistent among pools of individuals when quantifying the same selective mechanism. Furthermore, sexual selection based on $\beta^{\prime}$ fitted previously reported patterns of sexual selection and the common lizard's mating system. This was not the case when using $I$ and $\beta_{s s}$. Thus, estimates of the intensity of sexual selection using different indices, fitness proxies, and pools of individuals are not congruent with more pronounced differences in $I$ and $\beta_{\text {ss. }}$. These results indicate that metaanalyses of the intensity of sexual selection should take into account the index of sexual selection, the pool of individuals, and the fitness proxy used.

\section{Acknowledgments}

We thank T. van Dooren and three anonymous reviewers for comments that helped improve the manuscript. We also thank J. Cote, M.-L. Jarzat, and D. Mersch for assistance with population monitoring and O. Howard and B. Milá for English correction. Our research was funded by the French Ministry of Education and Research (ACI "Jeunes Chercheurs 2001"), the French Ministry of Environment (ACI "Invasions Biologiques"), the Federal Office of Education and Science (BBW grant 01.0254 to P.S.F.), the Swiss National Science Foundation (PPOOP3_128375), and the Spanish Ministry of Education and Science (CGL2005-01187 and CGL2008-01522/BOS).

\section{Literature Cited}

Ahnesjö, I., C. Kvarnemo, and S. Merilaita. 2001. Using potential reproductive rates to predict mating competition among individuals qualified to mate. Behavioral Ecology 12:397-401.

Andersson, M. 1994. Sexual selection. Princeton University Press, Princeton, NJ.

Andersson, M., and Y. Iwasa. 1996. Sexual selection. Trends in Ecology \& Evolution 11:A53-A58. 
Arnold, S. J., and D. Duvall. 1994. Animal mating systems: a synthesis based on selection theory. American Naturalist 143:317-348.

Bateman, A. J. 1948. Intra-sexual selection in Drosophila. Heredity 2:349-368.

Bauwens, D., and R. F. Verheyen. 1987. Variation of reproductive traits in a population of the lizard Lacerta vivipara. Holarctic Ecology 10:120-127.

Boudjemadi, K., O. Martin, J. C. Simon, and A. Estoup. 1999. Development and cross-species comparison of microsatellite markers in two lizard species, Lacerta vivipara and Podarcis muralis. Molecular Ecology 8:518-520.

Carranza, J. 2009. Defining sexual selection as sex-dependent selection. Animal Behaviour 77:749-751.

Clutton-Brock, T. H., and G. A. Parker. 1992. Potential reproductive rates and the operation of sexual selection. Quarterly Review of Biology 67:437-456.

Clutton-Brock, T. H., and A. C. J. Vincent. 1991. Sexual selection and the potential reproductive rates of males and females. Nature 351:58-60.

Crow, J. F. 1958. Some possibilities for measuring selection intensities in man. Human Biology 30:1-13.

Darwin, C. 1871. The descent of man, and selection in relation to sex. J. Murray, London.

Doherty, P. F., G. Sorci, J. A. Royle, J. E. Hines, J. D. Nichols, and T. Boulinier. 2003. Sexual selection affects local extinction and turnover in bird communities. Proceedings of the National Academy of Sciences of the USA 100:5858-5862.

Eberhard, W. G. 1996. Female control: sexual selection by cryptic female choice. Princeton University Press, Princeton, NJ.

Emlen, D. J., and L. W. Oring. 1977. Ecology, sexual selection, and the evolution of mating systems. Science 197:215-223.

Fitze, P. S., and J.-F. Le Galliard. 2008. Operational sex ratio, sexual conflict, and the intensity of sexual selection. Ecology Letters 11: 432-439.

Fitze, P. S., J.-F. Le Galliard, P. Federici, M. Richard, and J. Clobert. 2005. Conflict over multiple partner mating between males and females of polygynandrous common lizards. Evolution 59:24512459.

Fitze, P. S., J. Cote, J. P. Martínez-Rica, and J. Clobert. 2008. Determinants of male fitness: disentangling between intra- and intersexual selection. Journal of Evolutionary Biology 21:246-255.

Fitze, P. S., J. Cote, and J. Clobert. 2010. Mating order-dependent female mate choice in the polygynandrous common lizard Lacerta vivipara. Oecologia (Berlin) 162:331-341.

Grafen, A. 1988. On the uses of data on lifetime reproductive success. Pages 454-471 in T. H. Clutton-Brock, ed. Reproductive success. University of Chicago Press, Chicago.

Head, M. L., J. Hunt, M. D. Jennions, and R. Brooks. 2005. The indirect benefits of mating with attractive males outweigh the direct costs. PLoS Biology 3:289-294.

Hubbell, S. P., and L. K. Johnson. 1987. Environmental variance in lifetime mating success, mate choice, and sexual selection. American Naturalist 130:91-112.

Jones, A. G., G. Rosenqvist, A. Berglund, S. J. Arnold, and J. C. Avise. 2000. The Bateman gradient and the cause of sexual selection in a sex-role-reversed pipefish. Proceedings of the Royal Society B: Biological Sciences 267:677-680.

Jones, A. G., J. R. Arguello, and S. J. Arnold. 2004. Molecular parentage analysis in experimental newt populations: the response of mating system measures to variation in the operational sex ratio. American Naturalist 64:444-456.

Klug, H., J. Heuschele, M. D. Jennions, and H. Kokko. 2010. The mismeasurement of sexual selection. Journal of Evolutionary Biology 23:447-462.

Koenig, W. D., and S. S. Albano. 1986. On the measurement of sexual selection. American Naturalist 127:403-409.

Kokko, H., A. Mackenzie, J. D. Reynolds, J. Lindstrom, and W. J. Sutherland. 1999. Measures of inequality are not equal. American Naturalist 154:358-382.

Kvarnemo, C. 1996. Temperature affects operational sex ratio and intensity of male-male competition: experimental study of sand gobies, Pomatoschistus minutus. Behavioral Ecology 7:208-212.

Kvarnemo, C., and I. Ahnesjö. 1996. The dynamics of operational sex ratios and competition for mates. Trends in Ecology \& Evolution 11:404-408.

Laloi, D., M. Richard, J. Lecomte, M. Massot, and J. Clobert. 2004. Multiple paternity in clutches of common lizard Lacerta vivipara: data from microsatellite markers. Molecular Ecology 13:719-723.

Lande, R., and S. J. Arnold. 1983. The measurement of selection on correlated characters. Evolution 37:1210-1226.

Le Galliard, J.-F., R. Ferrière, and J. Clobert. 2003. Mother-offspring interactions affect natal dispersal in a lizard. Proceedings of the Royal Society B: Biological Sciences 270:1163-1169.

Le Galliard, J.-F., J. Clobert, and R. Ferrière. 2004. Physical performance and darwinian fitness in lizards. Nature 432:502-505.

Le Galliard, J.-F., R. Ferrière, and J. Clobert. 2005a. Juvenile growth and survival under dietary restriction: are males and females equal? Oikos 111:368-376.

Le Galliard, J.-F., P. S. Fitze, R. Ferrière, and J. Clobert. 2005b. Sex ratio bias, male aggression, and population collapse in lizards. Proceedings of the National Academy of Sciences of the USA 102: 18231-18236.

Le Galliard, J.-F., J. Cote, and P. S. Fitze. 2008. Lifetime and intergenerational fitness consequences of harmful male interactions for female lizards. Ecology 89:56-64.

Lyon, B. E., J. M. Eadie, and L. D. Hamilton. 1994. Parental choice selects for ornamental plumage in American coot chicks. Nature 371:240-243.

Marshall, T. C., J. Slate, L. E. B. Kruuk, and J. M. Pemberton. 1998. Statistical confidence for likelihood-based paternity inference in natural populations. Molecular Ecology 7:639-655.

Massot, M., J. Clobert, T. Pilorge, J. Lecomte, and R. Barbault. 1992. Density dependence in the common lizard: demographic consequences of a density manipulation. Ecology 73:1742-1756.

McLain, D. K. 1992. Population density and the intensity of sexual selection on body length in spatially or temporally restricted natural populations of a seed bug. Behavioral Ecology and Sociobiology 30:347-356.

Mills, S. C., A. Grapputo, E. Koskela, and T. Mappes. 2007. Quantitative measure of sexual selection with respect to the operational sex ratio: a comparison of selection indices. Proceedings of the Royal Society B: Biological Sciences 274:143-150.

Quinn, G. P., and M. J. Keough. 2002. Experimental design and data analysis for biologists. Cambridge University Press, Cambridge.

Richard, M., J. Lecomte, M. de Fraipont, and J. Clobert. 2005. Agespecific mating strategies and reproductive senescence. Molecular Ecology 14:3147-3155.

Shuker, D. M. 2010. Sexual selection: endless forms or tangled bank? Animal Behaviour 79:e11-e17. 
Shuster, S. M., and J. M. Wade. 2003. Mating systems and strategies. Princeton University Press, Princeton, NJ.

Sutherland, W. J. 1985. Measures of sexual selection. Oxford Surveys in Evolutionary Biology 1:90-101.

Vanpe, C., P. Kjellander, M. Galan, J. F. Cosson, S. Aulagnier, O. Liberg, and A. J. M. Hewison. 2008. Mating system, sexual dimorphism, and the opportunity for sexual selection in a territorial ungulate. Behavioral Ecology 19:309-316.

Wade, M. J. 1979. Sexual selection and variance in reproductive success. American Naturalist 114:742-747.

Wade, M. J., and S. J. Arnold. 1980. The intensity of sexual selection in relation to male sexual behaviour, female choice, and sperm precedence. Animal Behaviour 28:446-461.

Wade, M. J., and S. M. Shuster. 2004. Sexual selection: harem size and the variance in male reproductive success. American Naturalist 164:E83-E89.

Weir, L. K., J. W. A. Grant, and J. A. Hutchings. 2011. The influence of operational sex ratio on the intensity of competition for mates. American Naturalist 177:167-176.

Westneat, D. F. 2006. No evidence of current sexual selection on sexually dimorphic traits in a bird with high variance in mating success. American Naturalist 167:E171-E189.

Wolf, J. B., and M. J. Wade. 2001. On the assignment of fitness to parents and offspring: whose fitness is it and when does it matter? Journal of Evolutionary Biology 14:347-356.

Associate Editor: Suzanne H. Alonzo Editor: Ruth G. Shaw

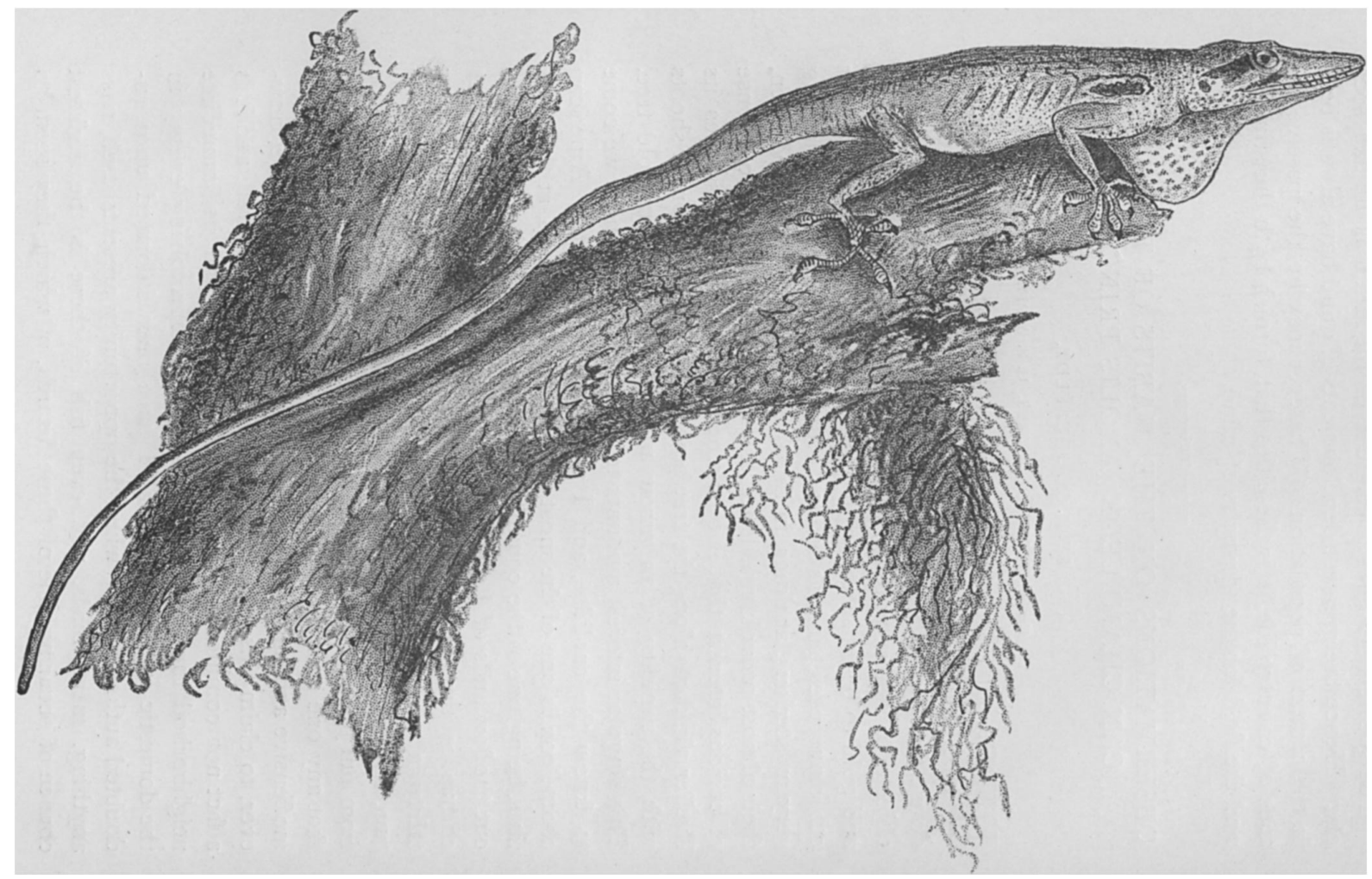

"Anolis principalis renders, by its constant destruction of those insects which infest the trees of our Southern cities, a great service, and that, too, in a very modest and unassuming way. In this respect, how much better they are than that miserable and noisy little foreigner, the so-called English sparrow." From "Observations on the Habits of the American Chameleon (Anolis principalis)" by R. W. Shufeldt (American Naturalist, 1883, 17:919-926). 